\title{
Performance Analysis of DSR and AODV Routing Protocols
}

\author{
Shakeel Ahmed
}

\begin{abstract}
An Ad-hoc network consists of wireless nodes dynamically forming a temporary network without the use of any existing network infrastructure or centralized administration. An Ad-hoc network needs to have routing protocols which can adopt dynamically changing topology. To accomplish this, a number of Ad hoc routing protocols have been proposed and implemented, which include Destination-Sequenced Distance-Vector (DSDV), Ad-hoc On-Demand Distance Vector Routing (AODV), Dynamic Source Routing (DSR) and Temporally Ordered Routing Algorithm (TORA). In this paper, we examine two on demand routing protocols DSR and AODV to analyze the performance differentials by varying network load, mobility and network size with the help of ns-2 simulator. Simulation results show that the DSR perfectly behaves with smaller networks with lower speed of nodes and AODV reveals more efficient use of bandwidth. The focus of our paper is on those approaches that are relevant to our work.
\end{abstract}

\section{Index Terms-AODV, DSR, MANET, NS-2.}

\section{INTRODUCTION}

Advances in wireless technology and greater user mobility have provided a major impetus towards development of an emerging class of self-organizing, rapidly deployable network architectures referred to as Ad-hoc networks. An Ad-hoc network is a collection of wireless mobile nodes dynamically forming a temporary network without relying on extraneous hardware. These networks are built, operated, and maintained by their constituent wireless nodes. These nodes generally have a limited transmission range and, so, each node seeks the assistance of its neighboring nodes in forwarding packets where mobile access to a wired network is either ineffective or impossible. Potential applications for this class of network include instant network infrastructure to support collaborative computing in temporary or mobile environments, emergency rescue networks for disaster management, remote control of electrical appliance, communication systems such as IVC (Inter-Vehicle Communications). The nodes in an Ad hoc network generally have limited battery power and, so, reactive routing protocols endeavor to save power by discovering routes only when they are essentially.

Our intention is to study the performance of two dynamic routing protocols for Ad hoc networks - Dynamic Source Routing protocol (DSR) [1], and Ad Hoc On-Demand Distance Vector protocol (AODV) [2]. Both DSR and AODV

Manuscript received November 11, 2012; revised December 14, 2012.

Shakeel Ahmed is with Computer Science Department, College of Computer Science and Information Technology, King Faisal University, AlHasa, Saudi Arabia (e-mail: shakeel@kfu.edu.sa). share an interesting common characteristic - they both initiate routing activities on an "on demand" basis. This reactive nature of these protocols is a significant departure from more traditional proactive protocols. The key motivation behind the design of on-demand protocols is the reduction of the routing load. High routing load usually has a significant performance impact in low bandwidth wireless links. While DSR and AODV share the on-demand behavior in that they initiate routing activities only in the presence of data packets in need of a route, many of their routing mechanics are very different. In particular, DSR uses source routing, but AODV uses a table-driven routing framework and destination sequence numbers. DSR does not rely on any timer-based activities, but AODV does to a certain extent. One of our goals in this study is to extract the relative merits of these mechanisms. The motivation is that a better understanding of the relative merits will serve as a cornerstone for development of more effective routing protocols for mobile Ad hoc networks.

The rest of the paper is organized as follows. In section 2 we briefly review the DSR and AODV protocols. In Section 3, we present a detailed critique of the two protocols, focusing on the differences on their dynamic behaviors that can lead to performance differences. In section 4 we described the simulation environment, Section 5 performance comparison of the protocols. We draw our conclusions in Section 6 .

\section{DESCRIPTION OF DSR/AODV AND RELATED WORK}

\section{A. DSR(Dynamic Source Routing)}

DSR (Dynamic Source Routing) [1] is a reactive on-demand routing protocol for multi-hop wireless Ad hoc networks of mobile nodes. DSR uses source routing and protocol composed of two main mechanisms-'Route Discovery' and 'Route Maintenance', which works together entirely, on-demand. It works only when two nodes want to communicate with each other. Route Discovery and Route Maintenance are built to behave according to changes in the routes in use, adjusting them-selves when needed.

The protocol allows routing of packets to be loop free and also allows caching of routes in nodes for future use. DSR allows multiple routes to any destination, thus can lead easily to load balancing or increase robustness. In the source routing technique, a sender determines the exact sequence of nodes through which to propagate a packet. The list of intermediate nodes for routing is explicitly contained in the packet's header. In DSR, every mobile node in the network needs to maintain a route cache where it caches source routes that it has learned. When a host wants to send a packet to some other host, it first 
checks its route cache for a source route to the destination. In the case a route is found, the sender uses this route to propagate the packet. Otherwise the source node initiates the route discovery process.

Route discovery works by flooding the network with route request (RREQ) packets. Each node receiving a RREQ, rebroadcasts it, unless it is the destination or it has a route to the destination in its route cache. Such a node replies to the RREQ with a route reply (RREP) packet that is routed back to the original source. RREQ and RREP packets are also source routed. The RREQ builds up the path traversed so far.

The RREP route itself back to the source by traversing this path backwards. The route carried back by the RREP packet is cached at the source for future use. If any link on a source route is broken, the source node is notified using a route error (RERR) packet. The source removes any route using this link from its cache. A new route discovery process must be initiated by the source, if this route is still needed.

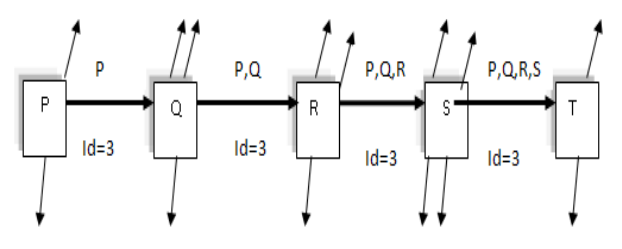

Fig. 1. Node $\mathrm{P}$ is the initiator and Node $\mathrm{T}$ is the target

\section{B. AODV (Ad hoc on-Demand Distance Vector)}

All tables should be numbered with Arabic numerals. Headings should be placed above tables, left justified. Leave one line space between the heading and the table. Only horizontal lines should be used within a table, to distinguish the column headings from the body of the table, and immediately above and below the table. Tables must be embedded into the text and not supplied separately. Below is an example which authors may find useful. AODV (Ad hoc on-demand distance vector) [2], is a purely reactive routing protocol; it offers low network utilization and uses destination sequence number to ensure loop freedom. In which each terminal does not need to keep a view of the whole network or a route to every other terminal. Nor does it need to periodically exchange route information with the neighbor terminals. When a mobile terminal has packets to send to a destination it need to discover and maintain a route to that destination terminal. In AODV, each terminal contains a route table for a destination and one entry per destination. A route table stores the following information: destination address and its sequence number, active neighbors for the route, hop count to the destination, and expiration time for the table. An important feature of AODV is that it uses a destination sequence number, which corresponds to a destination node that was requested by a routing sender node. The destination itself provides the number along with the route it has to take to reach from the request sender node up to the destination. If there are multiple routes from a request sender to a destination, the sender takes the route with a higher sequence number. The expiration time is updated each time the route is used. If this route has not been used for a specified period of time, it is discarded. This ensures that the Ad hoc network protocol remains loop-free.

According to the specification of AODV it includes an optimization technique to control the RREQ flood in the route discovery process. It uses an expanding ring search initially to discover routes to an unknown destination. In the expanding ring search, increasingly larger neighborhoods are searched to find the destination. The search is controlled by the TTL field in the IP header of the RREQ packets. If the route to a previously known destination is needed, the prior hop-wise distance is used to optimize the search.

\section{Simulation Results AND ANALYSIS}

The performance of the protocols depends on various interrelating adhered metrics. The most important parameters are Packet delivery rate, Mobility, Packet dropped and average end-to-end delay of data packets have been considered herein to draw an analytical observation using ns-2 simulator [4].

In this paper we use traffic and mobility model based on Continuous bit rate (CBR) traffic sources. Only 512-byte data packets are used. To change the offered load in the network the number of source-destination pairs and the packet sending rate in each pair is varied The mobility model uses the random waypoint model in a rectangular field. The field configurations used is: $500 \mathrm{~m} \times 500 \mathrm{~m}$ field with 10,2050 and 100 nodes. Here, each packet starts its journey from a random location to a random destination with a randomly chosen speed (uniformly distributed between 0-20 m/s). Simulations are run for 100 simulated seconds. Identical mobility and traffic scenarios are used across. The simulation parameters which have been considered for analyzing the performance comparison of two on-demand routing protocols is given below in Table I.

TABLE I: PARAMETERS USED IN EXPERIMENT SCENARIO

\begin{tabular}{l|l}
\hline \multicolumn{2}{l}{ Simulation Parameters } \\
\hline Protocols & AODV, DSR \\
Number of Mobile Nodes & $10,20,50,100$ \\
Simulation Area & $500 \mathrm{~m} \times 500 \mathrm{~m}$ \\
Simulation duration & 100 seconds \\
Mobility Model & Random waypoint \\
Traffic Type & Constant bit rate(CBR) \\
Packet Size & 512 bytes \\
Max Speed & $20 \mathrm{~m} / \mathrm{s}$ \\
\hline
\end{tabular}

Packet delivery rate: Packet delivery rate is the rate at which the data packets generated by the CBR sources and delivered to the destinations. The results shows that the rate of packet loss for each of the protocols AODV and DSR, simulated under the same conditions with 10 sources and at the same time comparing their rates of control packets used for the routing function.

Mobility: One of the major parameter of an Ad hoc network is Mobility. Since an Ad-hoc network is primarily characterized by its ever-changing topology, so mobility of nodes is an important consideration. Mobility of a node is a function of both speed and movement patterns. This simulation analysis is made from the Fig. 2 for 10 sources. First we analyze the first parameter Packet delivery ratio with respect varied Maximum speed of nodes. Fig. 2 shows the 
relative performance test result of the AODV and DSR routing protocols. All of the protocols deliver a greater percentage of the originated data packets when there is little node mobility, converging to $90 \%$ delivery ratio when there is no node motion. The On-demand protocol AODV performed particularly well, while DSR could not achieve good packet delivery ratio when moving more frequently.

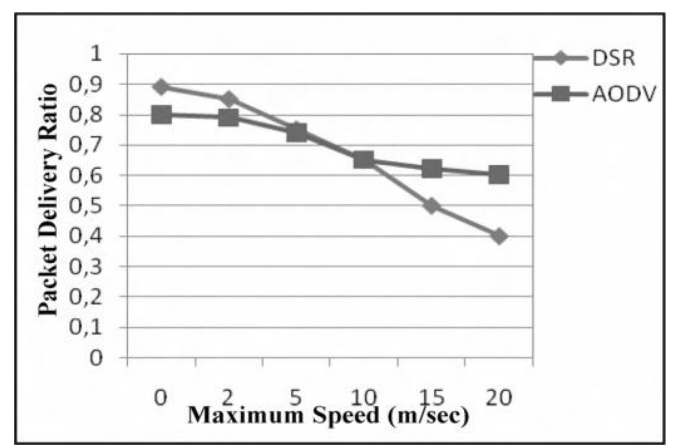

Fig. 2. Packet Delivery Ratio as function of Maximum Speed Mobility

End-To-End Delay: Fig. 3 shows the delay comparison of the two protocols. For on-demand-driven protocols, it is hard to say their performance relationship between the pause times. The curve jumped a lot with the pause time change. The second parameter Normalized End-To-End delay with varied pause times is analyzed and it is found that for DSR it is less when compared to AODV and we see that it is fairly stable even with increase number of sources. Further experiments should be done in order to make definitely conclusion.

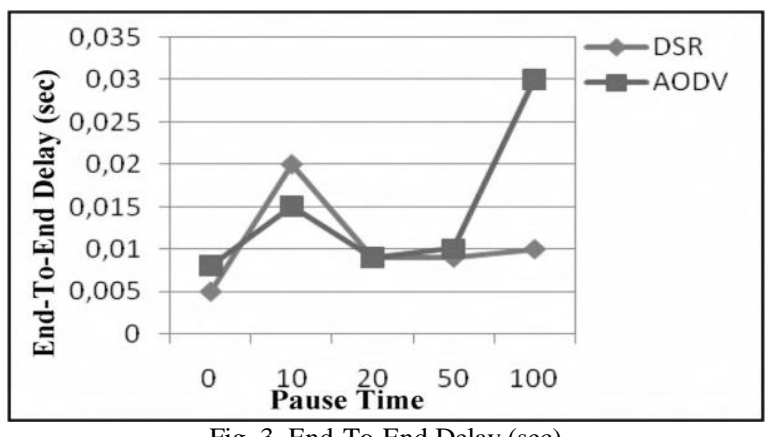

Fig. 3. End-To-End Delay (sec)

\section{CONCLUSION}

In this paper the basic actions related to the two routing protocols namely AODV and DSR were studied in detail.
On-demand driven protocols, as AODV, DSR, performed very well for packet delivery with fast movement and mobility rate. AODV seems to perform better than DSR on some situations. However, when mobility increases AODV has generally better performance. The On-demand protocol AODV performed particularly well, while DSR could not achieve good packet delivery ratio when moving more frequently. DSR is source routing protocol, which means that byte overhead in each packet can affect the total byte overhead when the load offered and size of the network increases. One advantage with source routing is that during route discovery operation it learns more routes. A combination of the protocols can be used for good result.

\section{REFERENCES}

[1] D. B. Johnson, D. A. Maltz, and Y. C. Hu, "The dynamic source routing protocol for mobile Ad Hoc networks (DSR)," Internet Draft, draft-ietf-manet-dsr- 09.txt, 15 April, 2003.

[2] C. Perkins, E. Belding-Royer, and S. Daas, "RFC 3561: Ad hoc on-demand distance vector routing," July 2003.

[3] Z. J. Haas and M. R. Pearlman, "The performance of query control schemes for the zone routing protocol," in Proc. of ACM SIGCOMM'98 Conference, pp. 167-177, Vancouver, Sept. 1998.

[4] Network Simulator-2 (NS2). [Online]. Available: http://www.isi.edu/nsnam/ns

[5] C. E. Perkins, E. M. Royer, S. R. Das and M. K. Marina, "Performance comparison of two on-demand routing protocols for $\mathrm{Ad} \mathrm{Hoc}$ networks," IEEE Personal communications, Feb. 2001.

[6] J. Broch, D. Maltz, D. Johnson, Y. Hu, and J. Jetsceva, "A performance comparison of multi-hop wireless Ad Hoc network routing protocols," Carnegie Mellon University, Pittsburgh.

[7] R. Misra and C. R. Manda, "Performance Comparison of AODV/DSR On-Demand Routing Protocols for Ad Hoc Networks in Constrained Situation," in Proc. IEEE ICPWC, 2005.

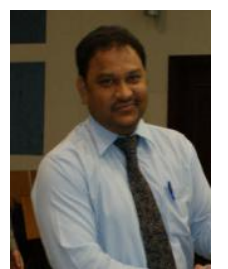

Shakeel Ahmed received his B.Sc (Computer. Science in 1997) from Kakatiya University and M.C.A (Master of Computer Applications in 2000) from M.K University, India. Currently he is working as a faculty member in the College of Computer Science and Information technology, King Faisal University, Saudi Arabia. His current research interests include Mobile Ad hoc networks, Software Engineering, Modeling of Systems using Formal Approaches. 